\title{
Terminating resuscitation for out-of-hospital cardiac arrest?
}

\author{
Reviewed by: Mohamed H. Azzam, MD;* Eddy Lang MDCM $;^{\dagger}$ Eli Segal MDCM ${ }^{\dagger}$
}

SeE RELATEd ARTICLE ON PAge 441

\author{
Clinical question \\ Can a clinical prediction rule for the termination of basic life support resuscitation for out-of- \\ hospital cardiac arrest (OHCA) identify those patients with little or no chance of survival? \\ Article chosen \\ Morrison LJ, Visentin LM, Kiss A, et al. Validation of a rule for termination of resuscitation in out- \\ of-hospital cardiac arrest. N Engl J Med 2006;355:478-487. \\ Study objective \\ To validate a previously derived clinical prediction rule for terminating futile resuscitation for OHCA.
}

\section{Background}

Despite advances in prehospital care, survival rates from out-of-hospital cardiac arrest (OHCA) remain dismally low. In many large cities in the United States, the overall survival for OHCA is as low as $1 \% .^{1-5}$ Yet, extensive resources are expended on resuscitation attempts and transport of patients who seem to have little or no chance of survival. This predicament has created the need to elucidate rational and evidence-based methods for providing prehospital resuscitation care.

\section{Population studied}

The study population consisted of consecutively enrolled adults ( $\geq 18$ years of age) who were treated for an outof-hospital arrest of presumed cardiac origin, as defined by Utstein criteria, ${ }^{6}$ between Jan. 1, 2002, and Jan. 30, 2004. Patients who had a cardiac arrest were evaluated and given basic life support (BLS) exclusively by emergency medical technicians (EMTs) trained in the use of an automated external defibrillator (AED). Patients were excluded if they received advanced cardiac life support (e.g., intubation and administration of intravenous fluids and medication), had a written or oral do-not-resuscitate order or had an arrest attributable to an obvious noncardiac cause (e.g., trauma or asphyxia).

\section{Study design}

The authors employed a prospective cohort study design for validating a clinical prediction rule using methods described by Wasson and colleagues ${ }^{7}$ and Laupacis and coauthors. ${ }^{8}$

All patients received BLS resuscitation consistent with the 2000 American Heart Association guidelines. ${ }^{9}$ Following transfer of care, the EMTs completed a data collection form that described clinical characteristics of the arrest as well as elements of the prediction rule.

For the purpose of validation, the rule was considered positive (i.e., termination of resuscitation was recommended) when all of the following 3 events occurred:

1. There was no return of spontaneous circulation.

*EM resident, McGill University, Montréal, Que., †Jewish General Hospital, Montréal, Que.

Submitted Dec. 6, 2007; Revised Mar. 28, 2008; Accepted Apr. 18, 2008

This article has not been peer reviewed.

CJEM 2008;10(5):474-7 
2. No shock was administered (i.e., there was no shockable rhythm detected by the AED).

3. The arrest was not witnessed by emergency medical services (EMS) personnel.

If any of these criteria was lacking, the rule was considered negative and continued resuscitative efforts and transportation to hospital were recommended. For the purpose of this study, however, all patients were transported to hospital and the findings of the rule had no bearing on EMT actions.

\section{Resuscitation algorithm}

The treatment algorithm used by the EMTs conformed to the American Heart Association guidelines and included the use of AED. After either successful defibrillation or the completion of this algorithm, the patient was transported rapidly to hospital and cardiopulmonary resuscitation continued if necessary. For the purpose of the study, the cardiac rhythm was analyzed only 3 times and no more than 3 shocks were delivered at each analysis to consider terminating resuscitation.

\section{Outcome measures}

\section{Primary outcome}

The performance characteristics of the termination of resuscitation rule, that is, the specificity and positive predictive value of the rule.

\section{Secondary outcome}

To evaluate whether 2 additional variables further increase the predictive power of the rule. These variables were

1. a response interval of more than 8 minutes.

2. a cardiac arrest not witnessed by a bystander.

Information about the patients' outcomes was obtained by the study coordinators $6-8$ months after the cardiac arrest. The outcomes were classified as follows.

Died:

- The patient was pronounced dead in the emergency department, or

- The patient died after admission to the hospital.

Survived:

- The patient was alive in the hospital at 6 months, or

- The patient was discharged home.

Cerebral performance was assessed using the Safar scale ${ }^{10}$ either at discharge or at 6-8 months postenrolment if the patient was still in the hospital. The estimated sample size was calculated to be able to predict a survival rate of $1 \%$ or less when the prediction rule recommended the termination of resuscitation. The authors defined it as a medically futile act if it yielded a benefit less than $1 \%$ of the time. ${ }^{11}$

\section{Results}

The study analyzed results on 1240 patients for whom complete datasets were available, representing $76.5 \%$ of all cardiac arrests encountered during the recruitment period. EMT noncompletion of data-collection forms was the most common reason for exclusion, though demographic data and survival rates were similar for enrolled and nonenrolled patients. The prediction rule performed with a specificity (i.e., the percentage of survivors in whom the rule was negative compared with all survivors) of $90.2 \%$ (95\% confidence interval [CI] 88.4-91.8), a sensitivity (i.e., the percentage of those deaths among patients in whom the rule was positive compared with all deaths) of $64.4 \%$ (95\% CI 61.6-67.0), a positive predictive value (i.e., the percentage of nonsurvivors among all patients with a positive test) of $99.5 \%$ (95\% CI 98.9-99.8) and a negative predictive value (i.e., the percentage of survivors among all patients who had a negative test) of $8 \%$ (95\% CI 6.6-9.7). Among the 776 patients for whom the rule suggested termination, 4 survived (a false positive rate $0.5 \%$ [95\% CI 0.1\%-0.9\%]) (Table 1). Of these 4 patients, 3 were discharged home or to a long-term care facility and were considered to have good cerebral performance (Cerebral Performance Category [CPC] 1) and 1 patient had severe cerebral disability (CPC 3).

The secondary outcomes of the study were as follows:

1. When the EMS response took more than 8 minutes, the prediction rule had a specificity of $97.6 \%$ (95\% CI 96.5-98.3) and a positive predictive value of $99.7 \%$

Table 1. Test characteristics of the clinical prediction rule

\begin{tabular}{|c|c|c|c|}
\hline \multirow[b]{2}{*}{$\begin{array}{l}\text { Action according to } \\
\text { prediction rule }\end{array}$} & \multicolumn{3}{|c|}{ Outcome } \\
\hline & Death & Survival & $\begin{array}{c}\text { No. of cardiac } \\
\text { arrests }\end{array}$ \\
\hline $\begin{array}{l}\text { Terminate BLS (test } \\
\text { positive) }\end{array}$ & 772 & 4 & 776 \\
\hline $\begin{array}{l}\text { Transport to ED (test } \\
\text { negative) }\end{array}$ & 427 & 37 & 464 \\
\hline Total & 1199 & 41 & 1240 \\
\hline $\begin{array}{l}\text { Survival rate when } \\
\text { termination } \\
\text { recommended by TOR } \\
\text { rule, } \%(95 \% \mathrm{Cl})\end{array}$ & \multicolumn{3}{|c|}{$0.5(0.1-0.9)$} \\
\hline $\begin{array}{l}\text { Sensitivity, } \\
\%(95 \% \mathrm{Cl})\end{array}$ & \multicolumn{3}{|c|}{$\begin{array}{c}64.4 \\
(61.6-67.0)\end{array}$} \\
\hline $\begin{array}{l}\text { Specificity, } \\
\%(95 \% \mathrm{Cl})\end{array}$ & \multicolumn{3}{|c|}{$\begin{array}{c}90.2 \\
(88.4-91.8)\end{array}$} \\
\hline $\begin{array}{l}\text { Positive predictive } \\
\text { value, } \%(95 \% \mathrm{Cl})\end{array}$ & \multicolumn{3}{|c|}{$\begin{array}{c}99.5 \\
(98.9-99.8)\end{array}$} \\
\hline $\begin{array}{l}\text { Negative predictive } \\
\text { value, } \%(95 \% \mathrm{Cl})\end{array}$ & \multicolumn{3}{|c|}{$\begin{array}{c}8.0 \\
(6.6-9.7)\end{array}$} \\
\hline
\end{tabular}

$\mathrm{BLS}=$ basic life support; $\mathrm{Cl}=$ confidence interval; $\mathrm{ED}=$ emergency department; $\mathrm{TOR}=$ termination of resuscitation. 
(95\% CI 99.2-99.9) (positive likelihood ratio [LR] 13.75 and negative LR 0.6). The survival rate based on this modification was $0.3 \%$ (95\% CI 0.0-1.7).

2. When the cardiac arrest was not witnessed by bystanders, both the specificity and the positive predictive value were $100 \%$ (95\% CI 99.6-100) (positive LR $\infty$ ). The survival rate among these patients was $0 \%(95 \%$ CI 0.0-1.0). (Note: both variables were analyzed separately in the post hoc analysis.)

Application of the original rule would have reduced the transport rate from $100 \%$ to $37.4 \%$ (this is derived from the inverse of the total rule positive rate of $62.6 \%$ [776/1240]). When one of the secondary outcomes was incorporated into the rule, this less sensitive but more specific version of the rule would have reduced the transport rate to $68.4 \%$ ( $>8 \mathrm{~min}$ response time) and $61.1 \%$ (unwitnessed) depending on which variable was used. The outcomes of all patients involved in the study are shown in Table 2.

\section{Study conclusion}

The authors validated a clinical prediction rule for the termination of resuscitation for OHCA with BLS that was found to have good specificity but only moderate sensitivity. Although it failed to identify 4 out of 776 (95\% CI $<1 \%$ ) patients who survived, most of whom had good cerebral performance, this was within the level of "medical futility" as defined by the authors a priori, as well as by some bioethicists. ${ }^{11}$ This rule may help EMS providers and directors implement protocols for the termination of BLS resuscitative efforts in patients experiencing OHCA.

\section{Commentary}

This study has validated a clinical prediction rule intended for use in OHCA by EMTs who are trained in BLS and the use of AEDs. Implementation of this rule would have resulted in a $62.6 \%$ reduction in the transport of patients at the expense of less than $1 \%$ of patients ( 4 in this study) who may have benefited from resuscitative efforts. This large reduction in transports may have a significant impact on resource consumption and reduced response times for other EMS calls. Moreover, it could also have a reduced risk for the community and EMS personnel in terms of transport safety. However, will any rule that carries in excess of a $0 \%$ miss rate be acceptable for implementation by EMS directors?

The question of whether this rule can be applied in its original form or with modification by additional variables may benefit from consideration of some Bayesian principles and an appreciation of the probability of survival from cardiac arrest in a given community. This can be achieved by using the rule's performance characteristics in the form of LRs and applying them with the use of a Fagan nomogram $^{12}$ against the pretest probability of death. The LR for any screening or diagnostic test expresses the relative likelihood that a given test result would be expected in a patient with a disorder of interest compared with the likelihood of the same result in a patient without the disorder.

Using the original rule's performance characteristics, we find a somewhat weak positive LR of 6.6 (positive test = rule recommended termination) and an equally unhelpful negative LR of 0.39 (negative test $=$ rule recommended transportation). The average survival rates for OHCA, including this study, range from $1 \%$ to $6.1 \%{ }^{4,13}$ Using the nomogram, we note that the termination of resuscitation rule performs quite differently at the extremes of these ranges (Fig. 1). In a setting with a low survival rate (1\%), the application of the original termination of resuscitation rule makes it very unlikely for patients who would have benefited from resuscitation to have been missed. However, if we assume a survival rate in the range of 5\%, the original rule carries just under a $1 \%$ risk of missing a patient who would have benefited from resuscitation.

Since a $1 \%$ miss rate may not be acceptable from a societal perspective, applying our modified version of the rule, which incorporates response times or a bystander witness, may be warranted in some settings. Applying the prediction rule in its original form led to a survival rate of $0.5 \%$ (95\% CI 0.1\%-0.9\%) among patients in whom termination of resuscitation was recommended. However, when one of the secondary outcome variables is added, the survival rate is reduced to $0 \%$ or $0.3 \%$, depending on the variable used, reflecting a postive LR of 13.75 or higher. Terminating resuscitative efforts for OHCA raises ethical concerns that

Table 2. Outcomes of 1240 reported cardiac arrests

\begin{tabular}{lc} 
Outcome & No. (and \%) of patients \\
\hline Death & $1199(97)$ \\
Pronounced in the ED & $1140(92)$ \\
After admission & $59(5)$ \\
Survival & $41(3)$ \\
In hospital 6 mo after cardiac & $2(<1)$ \\
arrest & \\
Discharged from hospital & $39(3)$ \\
Category of cerebral performance* & \\
Good performance & $29(71)$ \\
Moderate disability & $5(12)$ \\
Severe disability & $6(15)$ \\
Coma, vegetative state & $1(2)$ \\
\hline $\begin{array}{l}\text { ED }=\text { emergency department. } \\
\text { * Values for categories of cerebral performance were calculated as percentages } \\
\text { of the } 41 \text { survivors. }\end{array}$ \\
\hline
\end{tabular}


must be taken into consideration. Some stakeholders may feel that defining medical futility as $1 \%$ in cardiac arrest is unacceptable and the modified version may be deemed more appropriate. Medical futility has always been a concern in this type of research and most studies have failed to address it in a quantitative manner..$^{5,14,15}$

While other prediction rules for the termination of resuscitative efforts for OHCA have been validated, yielding similar results to this study, ${ }^{4,13}$ no other trial has reported as robust a dataset.

\section{Conclusion}

Evidence-based rules for termination of resuscitation for OHCA are a priority in prehospital care. This study represents the most comprehensive validation of a rule for termination of resuscitation and offers even more specific variants (fewer false positives) to consider. Adoption of the termination of resuscitation should be done taking into consideration local OHCA survival rates and with an explicit understanding of the ethical implications of its use.

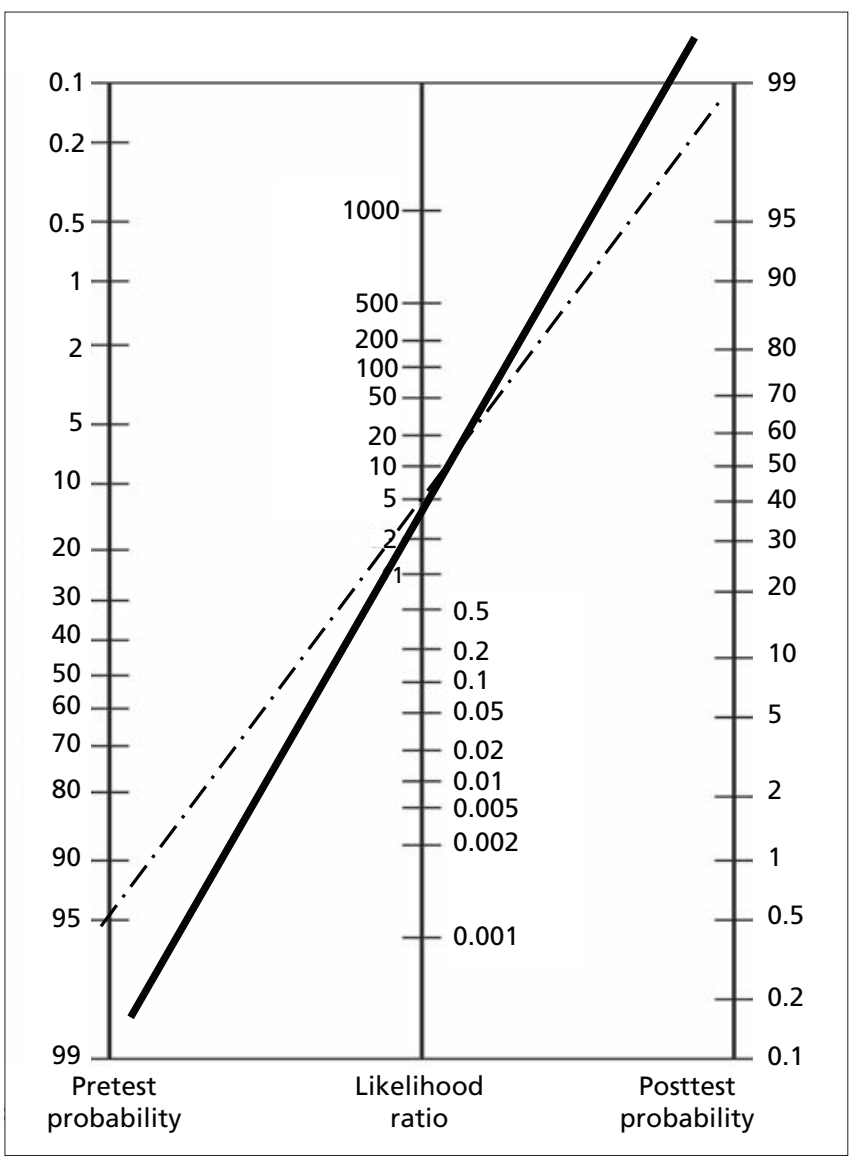

Fig. 1. Likelihood ratios for terminating out-of-hospital cardiac arrest. Survival rate $=5 \%$ (dashed line). Survival rate $=$ $1 \%$ (solid line).
Competing interests: None declared.

\section{References}

1. Ewy GA. Cardiac resuscitation - When is enough enough? N Engl J Med 2006;355:510-2.

2. ECC Committee, Subcommittees and Task Forces of the American Heart Association. 2005 American Heart Association guidelines for cardiopulmonary resuscitation and emergency cardiovascular care. Circulation 2005;112(24 Suppl):IV1-203.

3. Eckstein M, Stratton SJ, Chan LS. Cardiac arrest resuscitation evaluation in Los Angeles: CARE-LA. Ann Emerg Med 2005; 45:504-9.

4. Ong ME, Jaffey J, Stiell I, et al.; OPALS Study Group. Comparison of termination-of-resuscitation guidelines for basic life support: defibrillator providers in out-of-hospital cardiac arrest. Ann Emerg Med 2006;47:337-43.

5. Delbridge TR, Fosnocht DE, Garrison HG, et al. Field termination of unsuccessful out-of-hospital cardiac arrest resuscitation: acceptance by family members. Ann Emerg Med 1996;27:649-54.

6. Jacobs I, Nadkarni V, Bahr J, et al. Cardiac arrest and cardiopulmonary resuscitation outcome reports: update and simplification of the Utstein templates for resuscitation registries: a statement for healthcare professionals from a task force of the International Liaison Committee on Resuscitation (American Heart Association, European Resuscitation Council, Australian Resuscitation Council, New Zealand Resuscitation Council, Heart and Stroke Foundation of Canada, InterAmerican Heart Foundation, Resuscitation Councils of Southern Africa). Circulation 2004; 110:3385-97.

7. Wasson JH, Sox HC, Neff RK, et al. Clinical prediction rules: applications and methodological standards. N Engl J Med 1985; 313:793-9.

8. Laupacis A, Sekar N, Stiell IG. Clinical prediction rules: a review and suggested modifications of methodological standards. JAMA 1997;277:488-94.

9. ECC guideline. Part 3: Adult basic life support. Circulation 2000:102;I22-I59

10. Grenvik A, Safar P. Resuscitation after brain ischemia. In: Brain failure and resuscitation. New York (NY): Churchill Livingstone; 1981. p. 155-184.

11. Schneiderman LJ, Jecker NS, Jonsen AR. Medical futility: its meaning and ethical implications. Ann Intern Med 1990;112: 949-51.

12. Deeks JJ, Altman DG. Diagnostic test 4: likelihood ratio. BMJ 2004;329:168-9.

13. Ong ME, Tan EH, Ng FS. for the CARE study group. Comparison of termination-of-resuscitation guidelines for out-of-hospital cardiac arrest in Singapore EMS. Resuscitation 2007;75:244-51.

14. Edwardsen EA, Chiumento S, Davis E. Family perspective of medical care and grief support after field termination by emergency medical services personnel: a preliminary report. Prehosp Emerg Care 2002;6:440-4.

15. Soo LH, Gray D, Young T, et al. Resuscitation from out-ofhospital cardiac arrest: Is survival dependent on who is available at the scene? Heart 1999;81:47-52.

Correspondence to: Dr. Mohamed Hatem Azzam, 610 De La Mennais Ave., La Prairie QC J5R 2R1; dr_m_azzam2003@yahoo.com 